\title{
Chronic Abdominal Pain in General Practice
}

\author{
Cristina Maria Sabo Simona Grad Dan L. Dumitrascu
}

2nd Department of Internal Medicine, Iuliu Hatieganu University of Medicine and Pharmacy Cluj-Napoca, Cluj-Napoca, Romania

\section{Keywords}

Chronic abdominal pain · Diagnosis · General practice

\begin{abstract}
Background and Summary: Chronic abdominal pain is a challenging complaint for both primary care providers and gastroenterologists alike, due to a broad differential diagnosis and sometimes extensive and negative workup. In the absence of red flag features that herald more acute conditions, the majority of patients with chronic abdominal pain have a benign cause or a functional disorder (e.g., irritable bowel syndrome). The costs associated with a diagnostic workup are an expensive burden to health care. A systematic approach for evaluating patients and initiating a management plan are recommended in the primary care setting. Undiagnosed abdominal pain should be investigated starting with a detailed history and physical examination. Diagnostic investigations should be limited and adapted according to the clinical features, the alarm symptoms, and the symptom severity. This review will focus on the diagnostic tools which general practitioners utilize in the evaluation of chronic abdominal pain. Key Messages: The primary role of the general practitioner is to differentiate an organic disease
\end{abstract}

karger@karger.com

(c) 2021 S. Karger AG, Basel

www.karger.com/ddi

Karger! from a functional one, to refer to a specialist, or to provide treatment for the underlying cause of pain. The functional disorders should be considered after the organic pathology has been confidently excluded. Once a diagnosis of functional pain is established, repetitive testing is not recommended and the patient should be referred to receive psychological support (e.g., cognitive therapy) associated with available pharmacological therapeutic options.

(c) 2021 S. Karger AG, Base

\section{Introduction}

Pain is defined by the International Association for the Study of Pain (IASP) as "an unpleasant sensory and emotional experience associated with, or resembling that associated with, actual or potential tissue damage" [1].

Chronic pain is defined as a constant or recurrent pain that lasts for 3 months or more $[2,3]$. Chronic abdominal pain is mainly managed by general practitioners and it can be challenging, due to a broad differential diagnosis and extensive workup. A proportion of $24-35 \%$ of cases remain without a specific diagnosis following the primary care visit $[4,5]$. It is also an expensive burden to health 
care because this condition is often misdiagnosed and many general practitioners are unfamiliar with how to approach the diagnosis in a cost-effective manner.

Many gastrointestinal and systemic disorders may cause abdominal pain (Table 1 ). The primary care physician must consider the myriad of disorders, and narrow down the differential diagnoses, and drive further diagnostic testing when appropriate. Initial workup should include a detailed history, a physical examination, and diagnostic investigations tailored to the clinical presentation. If there are any alarm symptoms, the patient should be referred to a specialist because these could suggest organic disease. However, many patients with organic conditions do not have any alarm symptoms [6,7].

The leading causes of chronic abdominal pain are functional gastrointestinal disorders (FGIDs) or disorders of gut-brain interaction, such as functional dyspepsia (FD), irritable bowel syndrome (IBS), and centrally mediated abdominal pain syndrome (CAPS). These causes should be considered once organic pathology has been confidently excluded.

The aim of this review is to offer support to primary care physicians in a step-by-step investigation of the most common differential diagnoses, beginning with the key elements of the history and initial examination. Detailed patient history is vital for the differential diagnoses, especially as it informs a targeted physical examination, which can offer valuable clues regarding the etiology of the abdominal pain.

\section{Epidemiology}

Abdominal pain is the most common gastrointestinal symptom and one of the leading causes for both inpatient and outpatient visits as a recent US review suggests. International cross-sectional data estimate its prevalence between 22 and 25\%, with more women reporting abdominal pain than men (24 vs. $17 \%)[4,8]$.

Most patients with abdominal pain in the US tend to seek care with their primary care physician (84\%), while almost $40 \%$ see a gastroenterologist [9]. Among the primary care visits, $10 \%$ account for acute abdominal pain, while in the case of subacute or chronic abdominal pain, the most common diagnosis (13\% of visits) is IBS [10]. A proportion of $24-35 \%$ of cases remain without a specific diagnosis following the primary care visit $[4,5]$.

Abdominal pain was found to be the second most common location (after back pain); around 19\% of the Middle Eastern population were suffering from chronic
Table 1. Etiology of chronic abdominal pain

I. Abdominal wall pain

Herpes zoster

Postherpetic neuralgia

Segmental or intercostal neuralgia

Twelfth rib syndrome

Abdominal cutaneous nerve entrapment syndrome

II. Abdominal pain of visceral origin

Cardiac failure

Peptic ulcer disease

Gastritis and duodenitis

Gastroparesis

Carcinoma of the stomach

Post-gastric surgery syndrome

Carcinoma of the pancreas

Crohn's disease

Ulcerative colitis

Chronic constipation

Diverticular disease

Intestinal obstruction

Chronic mesenteric ischemia

Celiac artery syndrome

Abdominal adhesions

Colon cancer

Radiation enterocolitis

Chronic pancreatitis

Gallbladder disease

Post-cholecystectomy syndrome

Carcinoma of the liver or biliary system

Carcinoma of the kidney

Hernias

III. Abdominal pain syndromes of generalized diseases

Familial Mediterranean fever

Abdominal migraine

Intermittent acute porphyria

Hereditary coproporphyria

Variegate porphyria

IV. FGIDs or disorders of gut-brain interaction

FD

IBS

Functional abdominal pain syndrome

CAPS

Narcotic bowel syndrome

Gallbladder dyskinesia

Sphincter of Oddi dysfunction

Levator ani syndrome

Proctalgia fugax

Source: reproduced from [2]. FGIDs, functional gastrointestinal disorders; FD, functional dyspepsia; IBS, irritable bowel syndrome; CAPS, centrally mediated abdominal pain syndrome.

pain [11]. A meta-analysis of chronic pain in low- and middle-income countries estimated the prevalence of chronic abdominal pain at $17 \%$ of the general elderly population and found that chronic pain is specifically associ- 
ated with the female gender, advanced age, multiple pain sites, mood and psychosomatic disorders and is predictive of increased health-care costs $[12,13]$.

As far as the general age distribution of patients with chronic abdominal pain is concerned, data from the UK state that almost a third of cases occur in people under 20 years old, with a steady decrease in prevalence toward the old age [4].

Overall, the leading causes of chronic abdominal pain are FGIDs or disorders of gut-brain interaction. According to the Rome Foundation Global Study, $>40 \%$ of the global population meets the diagnosis criteria for at least one of the 22 syndromes comprised by FGIDs, and the most affected age-group is 18-39 [14].

The most common FGIDs responsible for chronic abdominal pain and their respective global prevalence are IBS (1-4\%), FD (2-7\%), functional constipation (7-11\%), and unspecified bowel disorder (0-11\%). A less frequent cause of FGID is CAPS (formerly known as functional abdominal pain syndrome [FAPS]). The prevalence of FAPS ranges between 0.5 and $1.7 \%$ and, similar to the general demographic distribution of FGIDs, is up to twice more frequent in women and in the 35-44 age-group $[15,16]$. There is an increased geographic heterogeneity among FGID rates, but smoking was identified as a consistent risk factor for this group of diseases, which also shows an association with other chronic pain syndromes [17].

In addition to visceral and functional abdominal pain, an often overlooked etiology is chronic abdominal wall pain (CAWP), responsible for $2-3 \%$ of cases of chronic abdominal pain. The prevalence of CAWP can rise up to $30 \%$ in cases with no identifiable organic cause, and it is 4 times more frequent in women, peaking in incidence between ages 30-50. The most common etiology of CAWP is abdominal cutaneous nerve entrapment syndrome, accounting for $10-30 \%$ of cases of chronic abdominal pain $[18,19]$.

\section{Etiology}

Chronic abdominal pain can arise from gastrointestinal disease and extraintestinal conditions involving the genitourinary tract, abdominal wall, thorax, and spine and often results in significant declines in function and quality of life.

According to the IASP taxonomy, chronic abdominal pain can be classified into abdominal wall pain, abdominal pain of visceral origin, abdominal pain syndromes of generalized diseases, and FGIDs (Table 1) [20].
Chronic abdominal pain can be roughly divided into visceral, somatosensory, and functional pain. While visceral pain typically originates from deep, internal abdominal structures, somatic pain originates from nociceptors in superficial tissues (i.e., skin) or the musculoskeletal system (i.e., bones, ligaments, and muscles) and functional pain relates primarily to visceral or central hypersensitivity [21].

Visceral pain is transmitted to the brain via vagal, thoracolumbar, and lumbosacral afferent nerves. Abdominal viscera receive dual innervation from the afferent and efferent system [22]. Visceral receptors are located on the serosal surfaces, within the mesentery, and in the walls of hollow viscera. Their stimuli are primarily chemical, while visceral nociceptors are sensitive to inflammatory mediators and are activated by distension, pressure, and ischemia [23].

Chronic abdominal pain due to true visceral pain is diffuse and poorly localizable with marked accompanying neurovegetative symptoms: nausea, vomiting, sweating, and emotional reactions such as distress and anxiety. The pain intensity is not necessarily fully correlated with the severity of the disease (e.g., mild/no pain in colon cancer, severe pain when passing a stool in IBS), and it is not always linked to injury (functional disorders) [21]. Chronic abdominal visceral pain may result from the following: (a) persistent inflammation (pancreatitis, cholecystitis, and inflammatory bowel disease); (b) vascular mechanisms: ischemia due to atherosclerosis or vasoconstriction (mesenteric ischemia); and (c) mechanical factors: obstruction of flow and distension of the organ (kidney stones and bile duct stones).

Chronic abdominal pain of musculoskeletal origin is sharp, localized to a small area (usually $<2 \mathrm{~cm}$ ), related to movement, and not correlated to eating and bowel function. Up to $10 \%$ of patients presenting chronic abdominal pain are diagnosed with CAWP, and it is most commonly caused by cutaneous nerve entrapment syndrome [24], which appears due to pressure from an intra- or extraabdominal lesion, or a localized process such as fibrosis or edema [25]. CAWP may also be caused by postsurgical pain, radiculopathy, diabetic neuropathy, or postherpetic neuralgia.

The leading causes of chronic abdominal pain are FGIDs or disorders of gut-brain interaction, such as FD, IBS, CAPS, and narcotic bowel syndrome. Extensive gastrointestinal evaluations often fail to show any organic cause. The pathogenesis of pain in FD and IBS is attributed to visceral hypersensitivity, through sensitization of peripheral afferent receptors or spinal dorsal horn neu- 
Table 2. Locations and characteristics of common causes of chronic abdominal pain

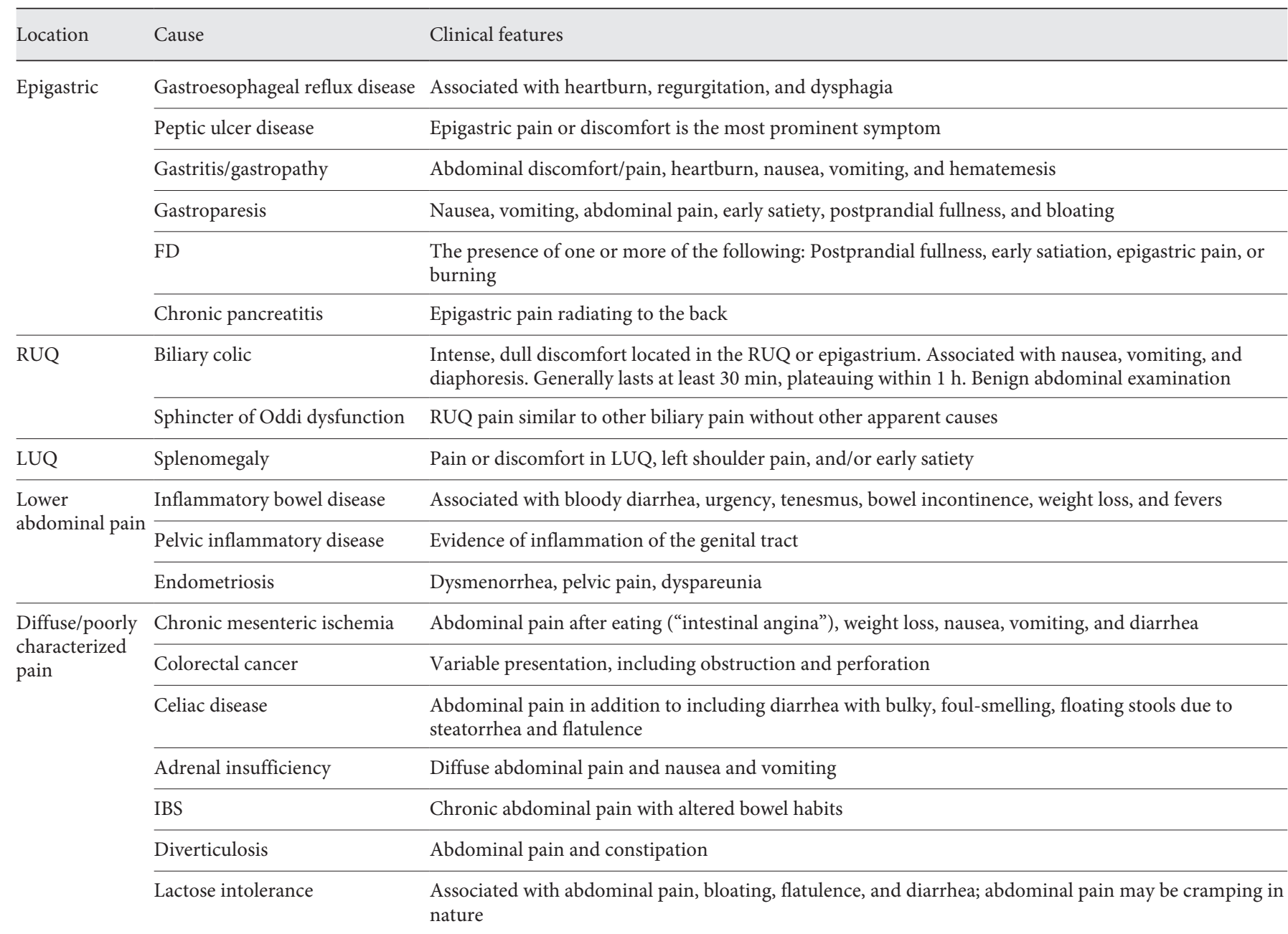

RUQ, right upper quadrant; LUQ, left upper quadrant; IBS, irritable bowel syndrome.

rons, alterations in descending modulation, or central amplification $[23,26]$. However, pain in CAPS is characterized by a strong central component and is relatively correlated with motility disturbance or visceral hypersensitivity [27].

\section{Clinical Presentation and Differential Diagnosis}

The evaluation of chronic abdominal pain requires an understanding of the possible mechanisms liable for pain, a broad differential of common causes (Table 1), and the recognition of the typical patterns and clinical presentations.

Chronic Abdominal Pain

\section{History}

The first step in managing a patient with chronic abdominal pain is to get a detailed history and the physical examination, even if these have a relatively low sensitivity and specificity in diagnosing the different causes of abdominal pain [28]. The abdominal pain characteristics, such as site, radiation, onset, the evolution of time, character, exacerbating/ameliorating factors, associations, and severity (SOCRATES [mnemonics]) can offer clues to the diagnosis (Table 2). The site and radiation of abdominal pain helps narrow down the differential diagnosis because the pain syndromes typically have characteristic locations. Upper abdominal pain can arise from biliary, pancreatic, gastric, and duodenal pathology. 
Table 3. Alarm symptoms or signs (termed red flags)

Onset of symptoms after 60

Unintended weight loss $>5 \%$

Loss of appetite

GI bleeding/iron deficiency anemia

Persistent vomiting

Family history of colorectal cancer or gastric cancer or inflammatory bowel disease

Mid-abdominal pain can be caused by the small bowel (e.g., Crohn's disease, celiac disease, partial small bowel obstruction, and chronic mesenteric ischemia). Lower abdominal pain arises from the colon (e.g., IBS and colitis), bladder, or reproductive organs. It is also important to establish the duration and the pattern of the pain (intermittent vs. constant) and the relationship to meals or bowel movements. Typically, the pain caused by peptic ulcer disease, chronic mesenteric ischemia, or biliary and pancreatic pathologies worsens after the meal, while in IBS pain is relieved with bowel movements. Chronic abdominal pain is defined as a constant or intermittent pain occurring for $>3$ months while acute abdominal pain is when the pain has been occurring for up to several days; subacute abdominal pain can span from several days up to 3 months [1]. Intermittent pain can have many causes but constant abdominal pain results only from a few gastrointestinal etiologies, such as chronic pancreatitis, malignancy, abscess, and psychiatric causes. One should probe for coexisting symptoms such as nausea, vomiting, diarrhea, blood in stools and systemic symptoms such as fever or rash. It is also important to review the past medical history such as comorbidities and medication use, especially opiate, nonsteroidal anti-inflammatory drugs (NSAIDs) as it can also aid in gauging the cause of the abdominal pain.

The alarm symptoms or signs (termed red flags) (Table 3) such as fever, weight loss, night sweats, loss of appetite, or nocturnal awakening should never be overlooked because these could suggest organic disease. Many patients with organic disease do not have red flags [7].

An additional aspect to consider when taking the medical history of the patient with chronic abdominal pain is the biopsychosocial dimension of the disease. Environmental exposure and personal factors, such as genetic factors, early trauma, health care-seeking behaviors or abuse can be triggered by certain life events (infections, major loss, somatic illness, unresolved interpersonal difficulties, or substance abuse), resulting in the expression or heightening of bodily pain.

Known diagnoses of sickle cell trait or disease, familial Mediterranean fever, and porphyria should be ascertained.

\section{Physical Examination}

A careful physical examination, including vital signs, detailed abdominal exam, and rectal examination, is the next stage, and it is important to clarify pain location and radiation patterns and to rule out significant pathology (organomegaly, masses, and acute abdomen). In the event of an acute episode of chronic abdominal pain, it is imperative to quickly rule out the possibility of a surgical abdomen.

A detailed physical examination is recommended, as it can provide useful clues of a systemic disease: lack of moist mucous membranes (dehydration); conjunctiva pallor (anemia); icteric sclera (hepatobiliary disease); dermatitis herpetiformis (celiac disease); erythema nodosum, pyoderma gangrenosum, and Sweet syndrome (inflammatory bowel disease); acanthosis nigricans (underlying malignancy); sunken eyes, prominent clavicles, and temporal wasting (significant weight loss); costovertebral angle tenderness (renal pathology); and vitamin deficiency [6].

A complete abdominal examination includes inspection, auscultation, percussion, palpation, and rectal examination. On inspection, the surgical scars should be noted. Identification of a bruit on auscultation may indicate chronic mesenteric ischemia. Light and deep palpation is useful to localize pain in a specific quadrant of the abdomen, to check for masses, ascites, hernias, and organomegaly and to observe the patient's response to palpation as it can be helpful in differentiating functional from organic disease. A rectal examination can offer valuable information, such as active bleeding, mass, signs of constipation, pelvic floor dysfunction, or high anal resting tone [29].

The Carnett's sign is useful to distinguish CAWP (e.g., somatic abdominal pain) from deeper visceral pain [30]. The test has a diagnostic accuracy of more than $90 \%$ for chronic pain of the abdominal wall [31]. The test is positive if the area of pain, which is usually quite small $(\leq 2$ $\mathrm{cm}$ ), worsens with abdominal muscle contraction when we ask the patient to raise their head from the bed without using their arms. 


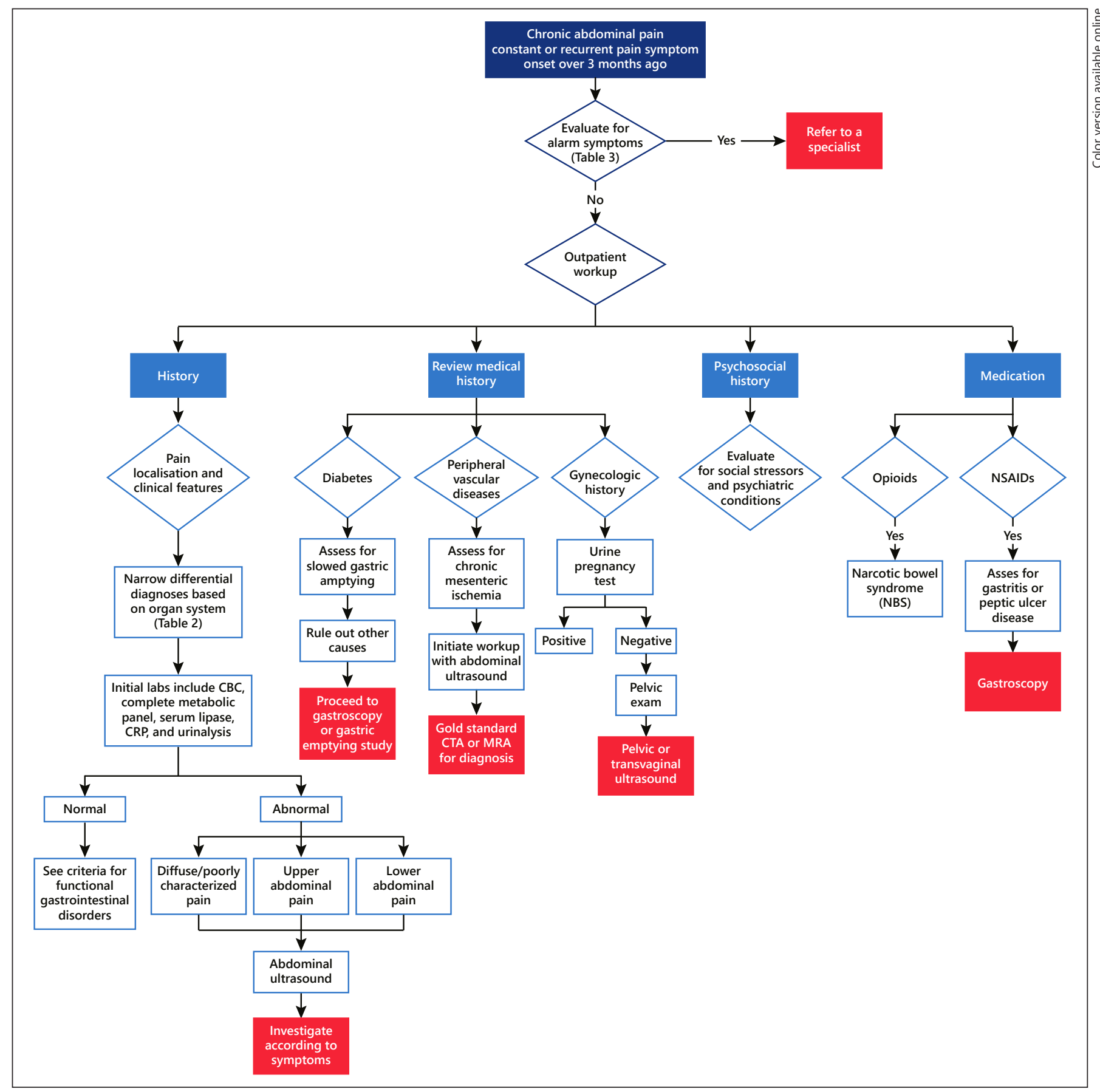

Fig. 1. Flowchart management of chronic abdominal pain in primary care. NSAIDS, nonsteroidal anti-inflammatory drugs; NBS, narcotic bowel syndrome.

\section{Diagnostic Workup in a Primary Care Setting}

We must keep in mind that not all abdominal pain is gastrointestinal in origin, and the workup of chronic abdominal pain should start with a detailed history and physical exam.
Begin by determining if there are any alarm symptoms to refer to a specialist. If alarm symptoms are absent, proceed to the outpatient workup, shown in Figure 1 [32].

A tailored initial workup, careful consideration of clinical features, symptom severity, or alarm features, as 
Table 4. Rome IV diagnostic criteria for FD

FD Diagnostic criteria (symptoms should have been active in the past 3 months, with onset at least 6 months before diagnosis)

One or more of the following: bothersome epigastric pain, bothersome epigastric burning, bothersome postprandial fullness, and bothersome early satiation

No evidence of structural disease (including at upper endoscopy) likely to explain these symptoms

Source: reproduced from [33]. FD, functional dyspepsia.

Table 6. Rome IV diagnostic criteria for FAPS

FAPS

\begin{tabular}{l} 
Must include all of the following* \\
Continuous or nearly continuous abdominal pain \\
\hline No or only occasional relationship of pain with \\
physiological events (e.g., eating, defecation, or menses) \\
Some loss of daily functioning \\
The pain is not feigned (e.g., malingering) \\
Insufficient symptoms to meet criteria for another \\
functional gastrointestinal disorder that would explain \\
the pain
\end{tabular}

Source: reproduced from [35]. FAPS, functional abdominal pain syndrome. ${ }^{*}$ Criteria fulfilled for the last 3 months with symptom onset at least 6 months before diagnosis.

well as prior therapeutic response, will allow the physician to limit unnecessary testing. Initial laboratory tests should include a full blood count, complete metabolic panel, serum lipase, C-reactive protein, and urinalysis. Other investigations should be based on individual patients, guided by the physician's suspicion. Diagnostic imaging is often not needed in patients with chronic abdominal pain, but when imaging is indicated, ultrasound, computed tomography scan, or MRI (including magnetic resonance cholangiopancreatography) should be chosen depending on suspected etiologies. If the alarm features are absent, symptoms are usually sufficient to diagnose FGIDs.

Once the diagnosis of functional gastrointestinal disorder is made, it can further be categorized by the Rome IV criteria into FD (Table 4) [33], IBS (Table 5) [34], FAPS (Table 6) [35], and CAPS (Table 7) [36].
Table 5. Rome IV diagnostic criteria $^{\text {a }}$ for IBS

IBS Recurrent abdominal pain on average at least 1 day/week in the last 3 months associated with 2 or more of the following

1. Can increase related to defecation

2. Associated with a change in frequency of stool

3. Associated with a change in form (appearance) of stool

Source: reproduced from [34]. IBS, irritable bowel syndrome. ${ }^{a}$ Criteria fulfilled for the last 3 months with symptom onset at least 6 months prior to diagnosis.

Table 7. Diagnostic criteria ${ }^{a}$ for CAPS

CAPS Must include all of the following

Continuous or nearly continuous abdominal pain

No or only occasional relationship of pain with physiological events (e.g., eating, defecation, or menses) ${ }^{c}$

Pain limits some aspect of daily functioning ${ }^{d}$

The pain is not feigned

Pain is not explained by another structural or functional gastrointestinal disorder or other medical condition

Source: reproduced from [36]. CAPS, centrally mediated

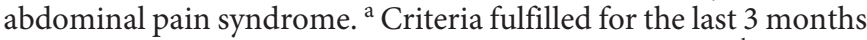
with symptom onset at least 6 months before diagnosis. ${ }^{b}$ CAPS is typically associated with psychiatric comorbidity, but there is no specific profile that can be used for diagnosis. ${ }^{c}$ Some degree of gastrointestinal dysfunction may be present. ${ }^{\mathrm{d}}$ Daily function could include impairments in work, intimacy, social/leisure, family life, and caregiving for self or others.

\section{Main Aspects and Challenges of Abdominal Pain in the Elderly}

The evaluation of the geriatric patients is often difficult because they wait much longer to seek medical attention and present with vague symptoms and have nonspecific findings on examination than younger patients, and also they have comorbidities and polypharmacy (e.g., $\beta$-blockers, steroids, NSAIDs, and opiates) which may alter their response to disease. The patient's ability to provide a history is frequently compromised by normal age-related decline in hearing and vision and cognitive impairments. Vital sign abnormalities such as fever, hypotension, and 
tachycardia may be absent due to concomitant medications (e.g., $\beta$-blockers and NSAIDs).

The most frequent causes of chronic abdominal pain in geriatric patients are peptic ulcer disease, biliary colic (calculous or acalculous), diverticular disease, chronic mesenteric ischemia, and colon cancer. It is also important to consider extra-abdominal abnormalities that may manifest as abdominal pain: congestive heart failure, genitourinary complaints (e.g., pyelonephritis, prostatitis, neurogenic bladder, and uterine prolapse), Herpes zoster, depression, or somatization [37].

Elderly patients more often had specific organic disease compared to younger patients and require the use of computed tomography scanning, often with intravenous contrast. The physician should use the glomerular filtration rate instead of serum $\mathrm{Cr}$ because it can be falsely elevated due to decreased muscle mass and protein intake, both very common in the elderly [38].

Regarding the treatment, it is important to assess the risk-benefit ratios and to select dose appropriately analgesic medication according to renal and liver insufficiency, dementia and fall risk, and patient tolerance or intolerance of opiates [39].

\section{Conclusions}

Chronic abdominal pain is a challenging diagnosis that requires frequently an extensive workup to identify functional or organic conditions by avoiding excessive investigation. In general practice, a detailed history and a careful physical exam should narrow down the list of differential diagnoses and limit unnecessary testing. Laboratory, endoscopic, and imaging examinations should be recommended in a rational cost-efficient approach.

\section{Conflict of Interest Statement}

The authors have no conflicts of interest to declare.

\section{Funding Sources}

The authors have no sources of funding to declare.

\section{Author Contributions}

S.C.M. drafted and revised the manuscript. G.S. reviewed and revised the manuscript. Both G.S. and D.L.D. approved the final draft submitted.

\section{References}

1 Raja SN, Carr DB, Cohen M, Finnerup NB, Flor H, Gibson S, et al. The revised international association for the study of pain definition of pain: concepts, challenges, and compromises. Pain. 2020 Sep 1;161(9):1976-82.

2 Treede RD, Rief W, Barke A, Aziz Q, Bennett MI, Benoliel R, et al. A classification of chronic pain for ICD-11. Pain. 2015;156(6):1003-7.

3 Bhatt A, Stevents T. Establishing diagnosis of chronic abdominal pain: gastroenterologist view. In: Kapural L, editor. Chronic abdominal pain: an evidence-based, comprehensive guide to clinical management. Springer Science+ Business Media New York; 2015. p. 25-31.

4 Wallander MA, Johansson S, Ruigómez A, García Rodríguez LA. Unspecified abdominal pain in primary care: The role of gastrointestinal morbidity. Int J Clin Pract. 2007;61(10): 1663-70.

5 BEACH Program, AIHW General Practice Statistics and Classification Unit. AIHW general practice statistics and classification unit. Presentations of abdominal pain in Australian general practice. Aust Fam Physician. 2004;33(7):2.

6 BMJ Best Practice. Evaluation of chronic abdominal pain. 2017 Nov;14.

7 Bharucha AE, Chakraborty S, Sletten CD. Common functional gastroenterological disorders associated with abdominal pain. Mayo Clin Proc. 2016;91(8):1118-32.
8 Sandler RS, Stewart WF, Liberman JN, Ricci JA, Zorich NL. Abdominal pain, bloating, and diarrhea in the United States: prevalence and impact. Dig Dis Sci. 2000 Jun;45(6):1166-71.

9 Lakhoo K, Almario CV, Khalil C, Spiegel BMR. Prevalence and characteristics of abdominal pain in the United States. Clin Gastroenterol Hepatol. 2020 [Online ahead of print].

10 Viniol A, Keunecke C, Biroga T, Stadje R, Dornieden K, Bösner S, et al. Studies of the symptom abdominal pain-a systematic review and meta-analysis. Fam Pract. 2014; 31(5):517-29.

11 El-Metwally A, Shaikh Q, Aldiab A, Al-Zahrani J, Al-Ghamdi S, Alrasheed AA, et al. The prevalence of chronic pain and its associated factors among Saudi Al-Kharj population: a cross sectional study. BMC Musculoskelet Disord. 2019;20(1):177-9.

12 Jackson T, Thomas S, Stabile V, Han X, Shotwell M, McQueen KA. Chronic pain without clear etiology in low- and middle-income countries: a narrative review. Anesth Analg. 2016;122(6):2028-39.

13 Jackson T, Thomas S, Stabile V, Shotwell M, Han X, McQueen K. A systematic review and meta-analysis of the global burden of chronic pain without clear etiology in low- and middle-income countries: trends in heterogeneous data and a proposal for new assessment methods. Anesth Analg. 2016;123(3):739-48.
14 Sperber AD, Bangdiwala SI, Drossman DA, Ghoshal UC, Simren M, Tack J, et al. Worldwide prevalence and burden of functional gastrointestinal disorders, results of rome foundation global study. Gastroenterology. 2021 Jan;160(1):99-114.e3.

15 Keefer L, Drossman DA, Guthrie E, Simrén $\mathrm{M}$, Tillisch K, Olden K, et al. Centrally mediated disorders of gastrointestinal pain. Gastroenterology. 2016 [Online ahead of print].

16 Sperber AD, Drossman DA. Review article: the functional abdominal pain syndrome. Aliment Pharmacol Ther. 2011;33(5):514-24.

17 Black CJ, Drossman DA, Talley NJ, Ruddy J, Ford AC. Functional gastrointestinal disorders: advances in understanding and management. Lancet. 2020 Nov 21;396(10263):1664-74.

18 Koop H, Koprdova S, Schürmann C. Chronic abdominal wall pain. A poorly recognized clinical problem. Dtsch Arztebl Int. 2016; 113(4):51-7.

19 Lindsetmo RO, Stulberg J. Chronic abdominal wall pain-a diagnostic challenge for the surgeon. Am J Surg. 2009 Jul;198(1):129-34.

20 Aziz Q, Giamberardino MA, Barke A, Korwisi B, Baranowski AP, Wesselmann U, et al. IASP taskforce for the classification of chronic pain. The IASP classification of chronic pain for ICD-11: chronic secondary visceral pain. Pain. 2019 Jan;160(1):69-76. 
21 Tolba R, Shroll J, Kanu A, Rizk MK. The epidemiology of chronic abdominal pain. In: Kapural L, editor. Chronic abdominal pain: an evidence-based, comprehensive guide to clinical management. Springer Science+Business Media New York; 2015. p. 18-24.

22 Camilleri M. Management options for irritable bowel syndrome. Mayo Clin Proc. 2018; 93(12):1858-72.

23 Pichetshote N, Pimentel M. An approach to the patient with chronic undiagnosed abdominal pain. Am J Gastroenterol. 2019 May; 114(5):726-32.

24 Srinivasan R, Greenbaum DS. Chronic abdominal wall pain: a frequently overlooked problem. Practical approach to diagnosis and management. Am J Gastroenterol. 2002; 97(4):824-30.

25 Oor JE, Ünlü C, Hazebroek EJ. A systematic review of the treatment for abdominal cutaneous nerve entrapment syndrome. Am J Surg. 2016;212(1):165-74.
26 Farmer AD, Aziz Q. Mechanisms and management of functional abdominal pain. J R Soc Med. 2014;107(9):347-54.

27 Schmulson MJ, Drossman DA. What is new in Rome IV. J Neurogastroenterol Motil. 2017;23(2):151-63.

28 Yamamoto W, Kono H, Maekawa M, Fukui $\mathrm{T}$. The relationship between abdominal pain regions and specific diseases: an epidemiologic approach to clinical practice. J Epidemiol. 1997;7(1):27.

29 Talley NJ. How to do and interpret a rectal examination in gastroenterology. Am J Gastroenterol. 2008;103(4):820-2.

30 Carnett JB. Pain and tenderness of the abdominal wall. JAMA. 1934;102(5):345-8.

31 Greenbaum DS, Greenbaum RB, Joseph JG, Natale JE. Chronic abdominal wall pain. Diagnostic validity and costs. Dig Dis Sci. 1994; 39(9):1935-41.

32 Mendelson R. Imaging for chronic abdominal pain in adults. Aust Prescr. 2015 Apr;38(2): 49-54.

33 Ford AC, Mahadeva S, Carbone MF, Lacy BE, Talley NJ. Functional dyspepsia. Lancet. 2020 Nov 21;396(10263):1689-702.
34 Schmulson MJ, Drossman DA. What is new in Rome IV. J Neurogastroenterol Motil. 2017 Apr 30;23(2):151-63.

35 Clouse RE, Mayer EA, Aziz Q, Drossman DA, Dumitrascu DL, Mönnikes H, et al. Functional abdominal pain syndrome. Gastroenterology. 2006 Apr;130(5):1492-7.

36 Keefer L, Drossman DA, Guthrie E, Simrén $\mathrm{M}$, Tillisch K, Olden K, et al. Centrally mediated disorders of gastrointestinal pain. Gastroenterology. 2016 [Online ahead of print].

37 Magidson PD, Martinez JP. Abdominal pain in the geriatric patient. Emerg Med Clin North Am. 2016 Aug;34(3):559-74.

38 Dowling TC, Wang ES, Ferrucci L, Sorkin JD. Glomerular filtration rate equations overestimate creatinine clearance in older individuals enrolled in the Baltimore longitudinal study on aging: impact on renal drug dosing. Pharmacotherapy. 2013;33(9):912-21.

39 Leuthauser A, McVane B. Abdominal pain in the geriatric patient. Emerg Med Clin North Am. 2016 May;34(2):363-75. 